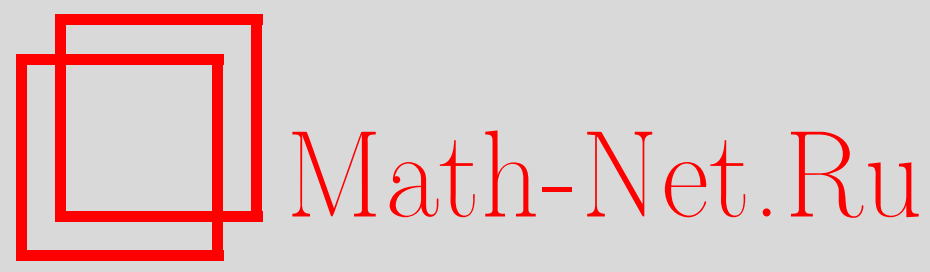

С. Ш. Галстян, Г. А. Карагулян, О расходимости почти всюду прямоугольных частичных сумм кратных рядов Фурье ограниченных функций, Матем. заметки, 1998, том 64, выпуск 1, 24-36

DOI: https://doi.org/10.4213/mzm1369

Использование Общероссийского математического портала Math-Net.Ru подразумевает, что вы прочитали и согласны с пользовательским соглашением http://www.mathnet.ru/rus/agreement

Параметры загрузки:

IP : 54.237 .206 .68

26 апреля 2023 г., 15:13:10

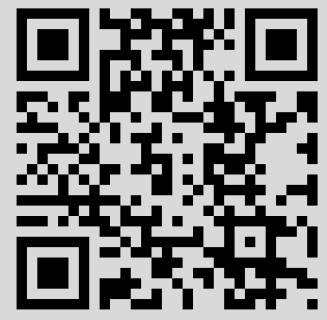




\section{О РАСХОДИМОСТИ ПОЧТИ ВСЮДУ ПРЯМОУГОЛЬНЫХ ЧАСТИЧНЫХ СУММ КРАТНЫХ РЯДОВ ФУРЬЕ ОГРАНИЧЕННЫХ ФУНКЦИЙ}

\section{С.Ш. Галстян, Г. А. Карагулян}

Установлены следующие результаты, являющиеся многомерными обобщениями известных теорем:

1) если функция $f \in C\left(\mathbb{T}^{m}\right)$ не имеет интервалов постоянства в $\mathbb{T}^{m}$, то существует гомеоморфизм $\varphi: \mathbb{T}^{m} \rightarrow \mathbb{T}^{m}$ такой, что ряд Фурье суперпозиции $F=f \circ \varphi$ расходится по прямоугольникам почти всюду;

2) для любой интегрируемой функции $f \in L^{1}\left(\mathbb{T}^{m}\right), c|f(x)| \geqslant \alpha>0, x \in \mathbb{T}^{m}$, сушествует функция знаков $\varepsilon(\boldsymbol{x})= \pm 1, \boldsymbol{x} \in \mathbb{T}^{m}$, такая, что ряд Фурье произведения $f(\boldsymbol{x}) \varepsilon(\boldsymbol{x})$ расходится по прямоугольникам почти всюду.

Библиография: 5 названий.

Мы рассматриваем кратные ряды

$$
\sum_{n \in \mathbb{Z}^{m}} a_{\boldsymbol{n}} e^{i \boldsymbol{n} \cdot \boldsymbol{x}}
$$

функций $f \in L^{1}\left(\mathbb{T}^{m}\right)$, где $\mathbb{T}^{m}-m$-мерньй тор, $\boldsymbol{n}=\left(n_{1}, \ldots, n_{m}\right), n_{i} \in \mathbb{Z}, \boldsymbol{x}=\left(x_{1}, \ldots\right.$, $\left.x_{m}\right) \in \mathbb{T}^{m}, \boldsymbol{n} \cdot \boldsymbol{x}=n_{1} x_{1}+\cdots+n_{m} x_{m}$.

В теории рядов Фурье известны многие результаты, в которых посредством естественных процедур, осуществляемых над функцией, получаются ряды Фурье с некоторьми свойствами. Подробньй обзор таких результатов в одномерном случае сделан А. М. Олевским в [1]. В частности, отметим следующий результат Г. Бора [2], доказанный комплексными методами: для любой вещественной функции $f \in C(\mathbb{T})$ существует гомеоморфизм $\varphi: \mathbb{T} \rightarrow \mathbb{T}$, такой, что ряд Фурье суперпозиции $F=f \circ \varphi$ сходится равномерно. А.А. Саакяном в [3], Ж.-П. Каханом и И. Кацнельсоном в [4] даны вещественные доказательства этой теоремы. С другой стороны, Олевский [1], решив проблему Н.Н. Лузина, доказал существование такой функции $f \in C(\mathbb{T})$, что каков бы ни был гомеоморфизм $\varphi: \mathbb{T} \rightarrow \mathbb{T}$, ряд Фурье суперпозиции $F=f \circ \varphi$ не является абсолютно сходящимся.

В кратном случае известен пример непрерьвной функции на $\mathbb{T}^{m}, m \geqslant 2$, построенный Ч. Фефферманом в [5], ряд Фурье которой расходится всюду по прямоугольникам. Саакян в [3] установил кратньй аналог теоремы Бора. А именно: для любой функции $f \in C\left(\mathbb{T}^{m}\right)$ существует гомеоморфизм $\varphi: \mathbb{T}^{m} \rightarrow \mathbb{T}^{m}$ такой, что ряд $f \circ \varphi$ сходится равномерно по прямоугольникам.

В работе получены результаты отрицательного характера. Они обобщают вьшеупомянутьй результат Феффермана. 
Tеорема 1. Пусть $f \in C\left(\mathbb{T}^{m}\right)$ есть некоторая функиия, не имеющая интервалов постоянства в $\mathbb{T}^{m}$. Тогда существует гомеоморфизм $\varphi: \mathbb{T}^{m} \rightarrow \mathbb{T}^{m}$ такой, что ряд Фурье суперпозичии $F=f \circ \varphi$ расходится почти всюду по прямоугольникам.

Tеорема 2. Для любой интегрируемой функиии $f \in L^{1}\left(\mathbb{T}^{m}\right) c|f(\boldsymbol{x})| \geqslant \alpha>0$, $x \in \mathbb{T}^{m}$, существует функиия знаков $\varepsilon(\boldsymbol{x}), \varepsilon(\boldsymbol{x})= \pm 1, \boldsymbol{x} \in \mathbb{T}^{m}$, такая, что ряд Фурье произведения $f(\boldsymbol{x}) \varepsilon(\boldsymbol{x})$ расходится почти всюду по прямоугольникам.

Пусть $f \in L^{1}\left(\mathbb{T}^{m}\right)$ - некоторая функция и $1 \leqslant a<b \leqslant \infty$ - произвольные числа. Обозначим $\boldsymbol{k}=\left(k_{1}, \ldots, k_{m}\right)$,

$$
\begin{gathered}
S_{[a, b]}^{*}(\boldsymbol{x}, f)=\max \left\{\left|S_{\boldsymbol{k}}(\boldsymbol{x}, f)\right|: \boldsymbol{k} \in \mathbb{Z}_{+}^{m}, a \leqslant k_{i} \leqslant b\right\}, \\
S^{*}(\boldsymbol{x}, f)=\sup _{\boldsymbol{k} \in \mathbb{Z}_{+}^{m}}\left|S_{\boldsymbol{k}}(\boldsymbol{x}, f)\right|, \quad \boldsymbol{x} \in \mathbb{T}^{m},
\end{gathered}
$$

где $S_{\boldsymbol{k}}(\boldsymbol{x}, f)$ - прямоугольные частичные суммы ряда $\Phi$ урье функции $f$. Обозначим также

$$
\Delta_{N}(x, f)=\left(\frac{N}{2 \pi}\right)^{m} \int_{\delta_{N}^{k}} f(t) d t \quad \text { при } \boldsymbol{x} \in \delta_{N}^{k},
$$

где $N$ - натуральное, а $\delta_{N}$ - семейство полуоткрытых кубов

$$
\begin{gathered}
\delta_{N}^{k}=\left[\frac{2 \pi k_{1}}{N}, \frac{\pi\left(k_{1}+1\right)}{N}\right) \times \cdots \times\left[\frac{2 \pi k_{m}}{N}, \frac{\pi\left(k_{m}+1\right)}{N}\right), \\
k=\left(k_{1}, \ldots k_{m}\right) \in I(N)=\left\{\boldsymbol{k} \in \mathbb{N}: 0 \leqslant k_{i}<N\right\} .
\end{gathered}
$$

Очевидно, семейство $\delta_{N}=\left\{\delta_{N}^{k}\right\}$ есть разбиение $\mathbb{T}^{m}$ на равные кубы со сторонами $2 \pi / N$.

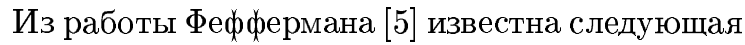

Лемма 1. Пусть $\lambda \in \mathbb{N}, 0<\varepsilon<\pi-$ некоторые числа $u$

$$
\begin{gathered}
\varphi_{\lambda}(\boldsymbol{x})=e^{i \lambda x_{1} x_{2}}, \quad \boldsymbol{x}=\left(x_{1}, \ldots, x_{m}\right) \in \mathbb{T}^{m}, \quad m \geqslant 2, \\
K_{\varepsilon}=\left\{\boldsymbol{x} \in \mathbb{T}^{m}:\left|x_{1}\right|>\varepsilon,\left|x_{2}\right|>\varepsilon\right\} .
\end{gathered}
$$

Тогда

$$
S_{[\varepsilon \lambda, 2 \pi \lambda]}^{*}\left(\boldsymbol{x}, \varphi_{\lambda}\right) \geqslant \xi(\varepsilon) \log \lambda \quad n p u \quad \boldsymbol{x} \in K_{\varepsilon},
$$

где $\xi(\varepsilon)>0$ есть постоянная, зависящая от $\varepsilon$ (основание логарифма равно 2).

$\mathrm{B}[5]$ эта лемма доказана при $\varepsilon=0.01$. Нам следует только отметить, что это доказательство без существенного изменения проходит также для произвольного $\varepsilon$.

Лемма 2. Пусть $f \in L^{1}\left(\mathbb{T}^{m}\right)$, a $E \subset \mathbb{T}^{m}$ - измеримое множество, причем

$$
|f(x)| \geqslant \alpha>0, \quad \Delta_{N}\left(x, \chi_{E}\right) \geqslant \beta>0, \quad x \in \mathbb{T}^{m},
$$

для некоторого натурального $N$. Тогда для любой функции $g(\boldsymbol{x}) \in L^{\infty}\left(\mathbb{T}^{m}\right) c$ условием

$$
\|g\|_{\infty} \leqslant \alpha \beta
$$

существует функиия $\varepsilon(\boldsymbol{x}), \boldsymbol{x} \in \mathbb{T}^{m}$, такая, что

$$
\varepsilon(\boldsymbol{x})=\left\{\begin{array}{ll} 
\pm 1, & \boldsymbol{x} \in E, \\
0, & \boldsymbol{x} \notin E,
\end{array} \quad \Delta_{N}(\boldsymbol{x}, f \varepsilon)=\Delta_{N}(\boldsymbol{x}, g), \quad \boldsymbol{x} \in \mathbb{T}^{m} .\right.
$$


ДоказАТЕЛьСтво. Для данного натурального $N$ и семейства чисел $h=\left\{h_{N}^{k}: k \in\right.$ $\left.I(N), 0 \leqslant h_{N}^{\boldsymbol{k}} \leqslant 2 \pi / N\right\}$ определим функцию $\varepsilon(\boldsymbol{x}, h), \boldsymbol{x} \in \mathbb{T}^{m}$. При $\boldsymbol{x} \in \delta_{N}^{\boldsymbol{k}}($ см. (2), (3)) возьмем

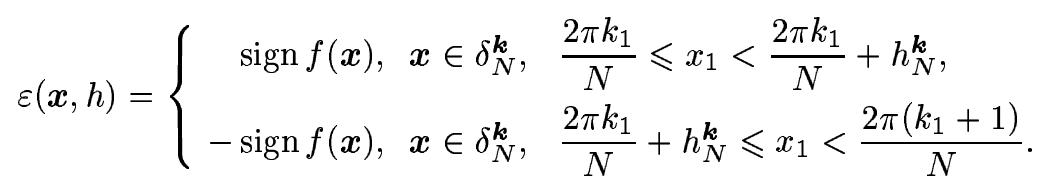

Из (7) следует, что

$$
\Delta_{N}\left(\boldsymbol{x}, f \chi_{E} \varepsilon\left(\cdot, h_{0}\right)\right) \leqslant-\alpha \beta, \quad \Delta_{N}\left(\boldsymbol{x}, f \chi_{E} \varepsilon\left(\cdot, h_{2 \pi / N}\right)\right) \geqslant \alpha \beta, \quad \boldsymbol{x} \in \mathbb{T}^{m},
$$

где $h_{0}$ есть семейство, состоящее из одних нулей, а $h_{2 \pi / N}$ состоит из $2 \pi / N$. Отсюда из соображений непрерьвности относительно каждого $h_{N}^{k}$ с учетом (1) и (8) следует, что для некоторого $h^{\prime}$

$$
\Delta_{N}\left(\boldsymbol{x}, f \chi_{E} \varepsilon\left(\cdot, h^{\prime}\right)\right)=\Delta_{N}(\boldsymbol{x}, g),
$$

что дает нам искомую функцию $\varepsilon(\boldsymbol{x})=\chi(\boldsymbol{x}) \varepsilon\left(\boldsymbol{x}, h^{\prime}\right)$.

Лемма 3. Пусть функиия $h \in L^{1}\left(\mathbb{T}^{m}\right)$ удовлетворяет условию

$$
\Delta_{N}(x, h)=0, \quad x \in \mathbb{T}^{m},
$$

для некоторого натурального $N$. Тогда справедливо неравенство

$$
\left|S_{n}(\boldsymbol{x}, h)\right| \leqslant \frac{n\left(\max m_{i}+1\right)^{m+1}}{(2 \pi)^{m-1} N}\|h\|_{L^{1}\left(\mathbb{T}^{m}\right)}, \quad n=\left(n_{1}, \ldots, n_{m}\right) .
$$

ДоКАЗАТЕЛЬСТво. Пусть

$$
D_{n}(\boldsymbol{x})=\frac{1}{2}+\sum_{k=1}^{n} \cos k x
$$

есть ядро Дирихле. Имеем

$$
\begin{aligned}
& \left|\prod_{i=1}^{m} D_{n_{i}}\left(x_{i}\right)-\prod_{i=1}^{m} D_{n_{i}}\left(y_{i}\right)\right| \\
& \quad=\left|\sum_{k=1}^{m}\left(\prod_{i=1}^{k-1} D_{n_{i}}\left(y_{i}\right) \prod_{i=k}^{m} D_{n_{i}}\left(x_{i}\right)-\prod_{i=1}^{k} D_{n_{i}}\left(y_{i}\right) \prod_{i=k+1}^{m} D_{n_{i}}\left(x_{i}\right)\right)\right| \\
& \quad=\left|\sum_{i=1}^{m} \prod_{i=1}^{k-1} D_{n_{i}}\left(y_{i}\right) \prod_{i=k+1}^{m} D_{n_{i}}\left(x_{i}\right)\left(D_{n_{i}}\left(x_{i}\right)-D_{n_{i}}\left(y_{i}\right)\right)\right| \\
& \quad \leqslant\left(\max n_{i}+\frac{1}{2}\right)^{m-1} \sum_{k=1}^{m}\left|D_{n_{i}}\left(x_{i}\right)-D_{n_{i}}\left(y_{i}\right)\right| \\
& \quad \leqslant\left(\max n_{i}+\frac{1}{2}\right)^{m-1} \sum_{k=1}^{m} \frac{m_{i}\left(m_{i}+1\right)}{2}\left|x_{i}-y_{i}\right| \\
& \quad \leqslant m\left(\max n_{i}+1\right)^{m+1} \max \left|x_{i}-y_{i}\right|
\end{aligned}
$$


где $\prod_{i=1}^{0}=\prod_{i=m+1}^{m}=1$. Отсюда в силу (9) имеем

$$
\begin{aligned}
\left|S_{n}(\boldsymbol{x}, h)\right| & =\frac{1}{(2 \pi)^{m}}\left|\int_{\mathbb{T}_{m}} \prod_{i=1}^{m} D_{m_{i}}\left(x_{i}+t_{i}\right) h(t) d t\right| \\
& =\left|\sum_{k \in \mathbb{Z}_{+}^{m}} \frac{1}{2 \pi} \int_{\delta_{N}}^{\boldsymbol{k}}\left(\prod_{i=1}^{m} D_{n_{i}}\left(x_{i}+t_{i}\right)-\prod_{i=1}^{m} D_{n_{i}}\left(x_{i}+\frac{2 \pi k_{i}}{N}\right)\right) h(t) d t\right| \\
& \leqslant \frac{m\left(\max n_{i}+1\right)^{m+1}}{N(2 \pi)^{m-1}}\|h\|_{L^{1}\left(\mathbb{T}^{m}\right)} .
\end{aligned}
$$

Лемма доказана.

ЛЕмма 4. Пусть $(X, \mu)$ есть конечное измеримое пространство, $a G_{i}, i=1,2$, .., $n$, - измеримые в нем множества такие, что

$$
\mu\left(G_{k} \backslash \bigcup_{i=1}^{k-1} G_{i}\right) \geqslant \alpha \mu\left(X \backslash \bigcup_{i=1}^{k-1} G_{i}\right), \quad k=1,2, \ldots, n
$$

при некотором $0<\alpha<1$. Тогда

$$
\mu\left(\bigcup_{i=1}^{n} G_{i}\right) \geqslant\left(1-(1-\alpha)^{n}\right) \mu(X)
$$

ДокАЗАТЕЛЬСТво. Докажем по индукции. Случай $n=1$ очевиден. Предположим, что (11) имеет место при $m=k$. Отсюда ввиду (10) имеем

$$
\begin{aligned}
\mu\left(\bigcup_{i=1}^{k+1} G_{i}\right) & =\mu\left(\bigcup_{i=1}^{k} G_{i}\right)+\mu\left(G_{k+1} \backslash \bigcup_{i=1}^{k} G_{i}\right) \\
& \geqslant(1-\alpha)\left(1-(1-\alpha)^{k}\right) \mu(X)+\alpha \mu(X)=\left(1-(1-\alpha)^{k+1}\right) \mu(X) .
\end{aligned}
$$

Лемма доказана.

ДОКАЗАТЕЛЬСТВо ТЕОРЕМЫ 2. ОбОЗначим

$$
Q(\delta, n)=\left\{x \in \mathbb{T}^{m}:\left\{\frac{n x_{i}}{2 \pi}\right\} \in[0, \delta), i=1, \ldots, m\right\},
$$

где $0<\delta<1$ и $n \in \mathbb{N}$. Очевидно, существует последовательность натуральных чисел $\left\{l_{k}\right\}_{k=1}^{\infty}$ такая, что

$$
\frac{l_{k}^{m+2}}{\sqrt{\log l_{k}}}>2 \sum_{i=k+1}^{\infty} \frac{1}{\sqrt{l_{i}}} .
$$

Будем рассматривать множества $Q_{k}=Q\left(\delta_{k}, n_{k}\right), k=1,2, \ldots$, где

$$
\delta_{k}=\frac{2}{\alpha \sqrt{\log l_{k}}}, \quad n_{k}=l_{k}^{m+2} .
$$


В силу (12) и (13) грубые оценки показьвают, что

$$
\Delta_{n_{k}} \boldsymbol{x}>\frac{1}{\alpha \sqrt{l_{k}}}, \quad \chi_{Q_{k} \backslash \bigcup_{i=k+1}^{\infty} Q_{i}}>\frac{1}{\alpha \sqrt{l_{k}}}, \quad k=1,2, \ldots .
$$

Далее по индукции мы построим функции $\varepsilon_{k}(\boldsymbol{x}), k=1,2, \ldots, \boldsymbol{x} \in \mathbb{T}^{m}$, такие, что

$$
\varepsilon_{k}(\boldsymbol{x})=\left\{\begin{aligned}
\pm 1, & x \in E_{k} \\
0, & x \notin \mathbb{T}^{m} \backslash E_{k}
\end{aligned}\right.
$$

где $E_{k}$ - множества, определенные равенствами

$$
E_{1}=\mathbb{T}^{m} \backslash \bigcup_{i=1}^{\infty} Q_{i}, \quad E_{k}=\bigcup_{i=N_{k}+1}^{N_{k+1}} Q_{i} \backslash \bigcup_{i=N_{k+1}+1}^{\infty} Q_{i}
$$

для каких-то натуральных чисел $N_{1}<N_{2}<\cdots$. От $\left\{\varepsilon_{k}\right\}_{k=1}^{\infty}$ будем требовать также выполнение следующих неравенств:

$$
\begin{gathered}
S_{\left[k, 2 \pi \lambda_{k}\right]}^{*}\left(x, \sum_{i=1}^{k} f \varepsilon_{i}\right)>k, \quad x \in G_{k} \subset \mathbb{T}^{m}, \\
S_{2 \pi \lambda_{k-1}}^{*}\left(x, f \varepsilon_{k}\right)<\frac{1}{2^{k}}, \quad x \in \mathbb{T}^{m},
\end{gathered}
$$

где $\lambda_{k} \rightarrow \infty-$ некоторая возрастающая последовательность положительных чисел, а для множеств $G_{k}$ имеем

$$
\left|G_{k} \backslash \bigcup_{i=[\sqrt{k}]^{2}}^{k-1} G_{i}\right|>\frac{1}{2}\left|\mathbb{T}^{m} \backslash \bigcup_{i=[\sqrt{k}]^{2}}^{k-1} G_{i}\right|-\frac{1}{2^{k}}, \quad k \geqslant 1
$$

Начальньй шаг индукции тривиален. Предположим, что (18) и (19) вьполняются при $k \leqslant p$. Обозначим

$$
R_{p+1}^{\prime}=\left\{x \in \mathbb{T}^{m}: S_{[p+1, \infty)}^{*}\left(x, \sum_{i=1}^{p} f \varepsilon_{i}\right)<\infty\right\}, \quad R_{p+1}^{\prime \prime}=\mathbb{T}^{m} \backslash R_{p+1}^{\prime}
$$

Очевидно, существуют множества $G_{p+1}^{\prime} \subset R_{p+1}^{\prime}, G_{p+1}^{\prime \prime} \subset R_{p+1}^{\prime \prime}$, числа $A_{p+1}>0$ и $\lambda_{p+1}^{\prime} \in \mathbb{N}, \lambda_{p+1}^{\prime}>2 \pi \lambda_{p}$, такие, что

$$
\begin{gathered}
\left|G_{p+1}^{\prime} \cup G_{p+1}^{\prime \prime}\right|>(2 \pi)^{m}-\frac{1}{2^{p+2}}, \\
S_{[p+1, \infty)}^{*}\left(x, \sum_{i=1}^{p} f \varepsilon_{i}\right)<A_{p+1}, \quad x \in A_{p+1}^{\prime}, \\
S_{\left[p+1, \lambda_{p+1}^{\prime}\right]}^{*}\left(x, \sum_{i=1}^{p} f \varepsilon_{i}\right)>p+2, \quad x \in G_{p+1}^{\prime \prime} .
\end{gathered}
$$


Далее возьмем натуральное $\lambda_{p+1}>\lambda_{p+1}^{\prime}$ так, чтобы (см. (6))

$$
\begin{aligned}
\left(\log \lambda_{p+1}\right)^{1 / 2} & >6 \max \left\{p+1, A_{p+1}\right\} \\
\lambda_{p+1} & >2^{p+2}(2 \pi)_{m}
\end{aligned}
$$

$2 \pi \lambda_{p+1}+1=l_{N_{p+1}}$ при некотором $N_{p+1}>N_{p}$, а также вьполнялись неравенства

$$
\begin{aligned}
& \left|\int_{\mathbb{T}^{m}} \operatorname{Re} \varphi_{\lambda_{p+1}} \cdot e^{i n \cdot \boldsymbol{x}} d \boldsymbol{x}\right|<\frac{1}{2^{p+2}\left(\lambda_{p}\right)^{2 m+1}}, \\
& \left|\int_{\mathbb{T}^{m}} \operatorname{Im} \varphi_{\lambda_{p+1}} \cdot e^{i n \cdot \boldsymbol{x}} d \boldsymbol{x}\right|<\frac{1}{2^{p+2}\left(\lambda_{p}\right)^{2 m+1}}
\end{aligned}
$$

при $\max \left|m_{i}\right| \leqslant \lambda_{p+1}^{\prime}$. Справедливость неравенств (26) при достаточно большом $\lambda_{p+1}$ следует из (4). Из (14), (15), (17) и (25) имеем

$$
\Delta_{\left(2 \pi \lambda_{p+1}+1\right)^{m+2}}\left(\boldsymbol{x}, \chi_{E_{p+1}}\right) \geqslant \Delta_{m_{p+1}}\left(\boldsymbol{x}, \chi_{Q_{N_{p+1}} \backslash \bigcup_{i \geqslant N_{p+1}} Q_{i}}\right) \geqslant \frac{1}{\alpha \sqrt{\lambda_{p+1}}} .
$$

Из (6) и (24) следует, что

$$
\max \left\{S_{\left[p+1 ; 2 \pi \lambda_{p+1}\right]}^{*}\left(x, \operatorname{Re} \varphi_{\lambda_{p+1}}\right), S_{\left[p+1 ; 2 \pi \lambda_{p+1}\right]}^{*}\left(x, \operatorname{Im} \varphi_{\lambda_{p+1}}\right)\right\} \geqslant \frac{1}{2} \xi\left(\varepsilon_{p, m}\right) \log \lambda_{p+1}
$$

при $\boldsymbol{x} \in K_{\varepsilon_{p, m}}$, где $\varepsilon_{p, m}=2^{-p-3}(2 \pi)^{1-m}$. Обозначим

$$
\begin{aligned}
H^{\prime} & =\left\{\boldsymbol{x}: S_{\left[p+1 ; 2 \pi \lambda_{p+1}\right]}^{*}\left(\boldsymbol{x}, \operatorname{Re} \varphi_{\lambda_{p+1}}\right) \geqslant \frac{1}{2} \xi\left(\varepsilon_{p, m}\right) \log \lambda_{p+1}\right\} \\
H^{\prime \prime} & =\left\{\boldsymbol{x}: S_{\left[p+1 ; 2 \pi \lambda_{p+1}\right]}^{*}\left(\boldsymbol{x}, \operatorname{Im} \varphi_{\lambda_{p+1}}\right) \geqslant \frac{1}{2} \xi\left(\varepsilon_{p, m}\right) \log \lambda_{p+1}\right\} .
\end{aligned}
$$

Из (5) и (28) имеем

$$
\left|K_{2^{-p-3}(2 \pi) 1-m}\right|>(2 \pi)^{m}-\frac{1}{2^{p+1}}, \quad H^{\prime} \cup H^{\prime \prime}=K_{\varepsilon_{p, m}} .
$$

Отсюда легко следует, что для одного из двух множеств $H^{\prime}$ или $H^{\prime \prime}$, которое обозначим через $H_{p+1}$, имеет место неравенство

$$
\left|H_{p+1} \backslash \bigcup_{i=[\sqrt{p+1}]^{2}}^{p} G_{i}\right|>\frac{1}{2}\left|\mathbb{T}^{m} \backslash \bigcup_{i=[\sqrt{p+1}]^{2}}^{p} G_{i}\right|-\frac{1}{2^{p+2}}
$$

Обозначим через $\psi_{p+1}$ ту из функций $\operatorname{Re} \varphi_{\lambda_{p+1}}$ и $\operatorname{Im} \varphi_{\lambda_{p+1}}$, которая участвует в определении $H_{p+1}$ (см. (29)). Тогда в силу неравенства (26) имеем

$$
\begin{gathered}
H_{p+1}=\left\{\boldsymbol{x}: S_{\left[p+1 ; 2 \pi \lambda_{p+1}\right]}^{*}\left(\boldsymbol{x}, \psi_{p+1}\right) \geqslant \frac{1}{2} \xi\left(\varepsilon_{p, m}\right) \log \lambda_{p+1}\right\} \\
\left|\int_{\mathbb{T}^{m}} \psi_{p+1}(\boldsymbol{x}) e^{i \boldsymbol{n} \cdot \boldsymbol{x}} d \boldsymbol{x}\right| \leqslant 2^{-p-3}\left(2 \lambda_{p+1}^{\prime}+1\right)^{m}
\end{gathered}
$$


при $\max \left|n_{i}\right| \leqslant \lambda_{p+1}^{\prime}$. В результате применения леммы 2, полагая

$$
N=\left(\lambda_{p+1}\right)^{m+1}, \quad \beta=\left(\log \lambda_{p+1}\right)^{-1 / 2} \alpha^{-1}, \quad g(\boldsymbol{x})=g_{p+1}(\boldsymbol{x})=\left(\log \lambda_{p+1}\right)^{-1 / 2} \psi_{p+1}(\boldsymbol{x})
$$

и учитьвая $(27)$, получим функцию $\varepsilon_{p+1}(\boldsymbol{x})$ вида $(16)($ где $k=p+1)$ такую, что

$$
\Delta_{\left(2 \pi \lambda_{p+1}+1\right)^{m+2}}\left(\boldsymbol{x}, f \varepsilon_{p+1}\right)=\Delta_{\left(2 \pi \lambda_{p+1}+1\right)^{m+2}}\left(\boldsymbol{x}, g_{p+1}\right) .
$$

Очевидно, можно предполагать $\|f\|_{L^{1}} \leqslant 1$. Далее, в силу (33) можно применить лемму 3 для $h=f \varepsilon_{p+1}-g_{p+1}, N=\left(2 \pi \lambda_{p+1}+1\right)^{m+2}$. С учетом $(24)$ при $\max n_{i} \leqslant\left(2 \pi \lambda_{p+1}+1\right)^{m+1}$ получим (см. (33))

$$
\begin{aligned}
\left|S_{n}\left(\boldsymbol{x}, f \varepsilon_{p+1}\right)-S_{n}\left(\boldsymbol{x}, g_{p+1}\right)\right| & =\left|S_{n}(\boldsymbol{x}, h)\right| \\
& \leqslant \frac{m}{(2 \pi)^{1-m}} \frac{\left(\max n_{i}+1\right)^{m+1}}{\left(2 \pi \lambda_{p+1}+1\right)^{m+2}}\left(\|f\|_{L^{1}}+1\right) \leqslant 2^{-p-2} .
\end{aligned}
$$

С другой стороны, в силу (33), (31) и (24) из леммы 1 следует, что

$$
\begin{aligned}
S_{\left[p+1 ; 2 \pi \lambda_{p+1}\right]}^{*}\left(x, g_{p+1}\right) & =\left(\log \lambda_{p+1}\right)^{-1 / 2} S_{\left[p+1,2 \pi \lambda_{p+1}\right]}^{*}\left(x, \psi_{p+1}\right) \\
& \geqslant \frac{1}{2} \xi\left(2^{-p-3}(2 \pi)^{1-m}\right)\left(\log \lambda_{p+1}\right)^{1 / 2} \quad \text { при } \quad x \in H_{p+1},
\end{aligned}
$$

и, следовательно, из $(22)$ и $(24)$ при $\boldsymbol{x} \in H_{p+1} \cap G_{p+1}^{\prime}$ имеем

$$
\begin{aligned}
S_{\left[p+1,2 \pi \lambda_{p+1}\right]}^{*}\left(x, \sum_{i=1}^{p+1} \varepsilon_{i} f\right) & \geqslant S_{\left[p+1,2 \pi \lambda_{p+1}\right]}^{*}\left(x, f \varepsilon_{p+1}\right)-S_{[p+1, \infty]}^{*}\left(x, \sum_{i=1}^{p} \varepsilon_{i} f\right) \\
& \geqslant \frac{1}{2} \xi\left(\frac{1}{2^{p+2}(2 \pi)^{m-1}}\right)\left(\log \lambda_{p+1}\right)^{1 / 2}-A_{p+1}-\frac{1}{2^{p+1}}>p+1 .
\end{aligned}
$$

Из (32) следует неравенство

$$
S_{\lambda_{p+1}^{\prime}}\left(\boldsymbol{x}, \psi_{p+1}\right) \leqslant \frac{1}{2^{p+2}}
$$

которое вместе с (33) и (34) дает

$$
S_{\lambda_{p+1}^{\prime}}\left(x, f \varepsilon_{p+1}\right) \leqslant \frac{1}{2^{p+1}}
$$

и, следовательно, если $\boldsymbol{x} \in G_{p+1}^{\prime \prime}$, то из (23) имеем

$$
\begin{aligned}
S_{\left[p+1,2 \pi \lambda_{p+1}\right]}^{*}\left(\boldsymbol{x}, \sum_{i=1}^{p+1} \varepsilon_{i} f\right) & \geqslant S_{\left[p+1, \lambda_{p+1}^{\prime}\right]}^{*}\left(\boldsymbol{x}, \sum_{i=1}^{p} \varepsilon_{i} f\right)-S_{\lambda_{p+1}^{\prime}}^{*}\left(\boldsymbol{x}, f \varepsilon_{p+1}\right) \\
& \geqslant p+2-\frac{1}{2^{p}}>p+1, \quad x \in G_{p+1}^{\prime \prime} .
\end{aligned}
$$


Из (35) и (37) получаем

$$
S_{\left[p+1,2 \pi \lambda_{p+1}\right]}^{*}\left(x, \sum_{i=1}^{p+1} \varepsilon_{i} f\right) \geqslant p+1, \quad x \in H_{p+1} \cap\left(G_{p+1}^{\prime} \cup G_{p+1}^{\prime \prime}\right) .
$$

Далее, обозначив $G_{p+1}=H_{p+1} \cap\left(G_{p+1}^{\prime} \cup G_{p+1}^{\prime \prime}\right)$, из (21) и (30) имеем (20) и (18) в случае $k=p+1$. Неравенство (19) (при $k=p+1$ ) немедленно следует из (36), если учесть, что $\lambda_{p+1}^{\prime}>2 \pi \lambda_{p}$.

Итак, построена последовательность функций $\left\{\varepsilon_{k}(\boldsymbol{x})\right\}$ со свойствами (16)-(20). Из (16) и (17) следует корректность определения функции

$$
\varepsilon(\boldsymbol{x})=\varepsilon_{k}(\boldsymbol{x}) \quad \text { при } \quad \boldsymbol{x} \in E_{k}, \quad k=1,2, \ldots,
$$

на $\mathbb{T}^{m}$. Из $(20)$ имеем

$$
\left|G_{k} \backslash \bigcup_{i=t^{2}}^{k-1} G_{i}\right|>\frac{1}{2}\left|\mathbb{T}^{m} \backslash \bigcup_{i=t^{2}}^{k-1} G_{i}\right|-\frac{1}{2^{t^{2}}} \quad \text { при } t^{2} \leqslant k<(t+1)^{2} .
$$

Покажем, что

$$
\left|\bigcup_{i=t^{2}}^{(t+1)^{2}-1} G_{i}\right|>\left(1-\left(\frac{3}{4}\right)^{2 t+1}\right)(2 \pi)^{m} .
$$

В том случае, когда существует $k_{0} \in\left[t^{2},(t+1)^{2}\right]$ с условием

$$
\left|\mathbb{T}^{m} \backslash \bigcup_{i=t^{2}}^{k_{0}-1} G_{i}\right|<\frac{4}{2^{t^{2}}}
$$

утверждение (39) следует очевидным образом. А в противном случае ввиду (38) имеем

$$
\left|G_{k} \backslash \bigcup_{i=t^{2}}^{k-1} G_{i}\right|>\frac{1}{2}\left|\mathbb{T}^{m} \backslash \bigcup_{i=t^{2}}^{k-1} G_{i}\right|-\frac{1}{2^{t^{2}}}>\frac{1}{4}\left|\mathbb{T}^{m} \backslash \bigcup_{i=t^{2}}^{k-1} G_{i}\right|
$$

и, следовательно, применив лемму 4, получаем (39), откуда следует, что для множества $G=\underline{\lim }_{k \rightarrow \infty} G_{k}$ вьполнено

$$
|G|=1
$$

С другой стороны, если $\boldsymbol{x} \in G$, то $\boldsymbol{x} \in G_{n_{k}}$ для некоторой последовательности $n_{k} \rightarrow \infty$. Отсюда благодаря (18) и (19) имеем

$$
\begin{aligned}
S_{\left[n_{k}, 2 \pi \lambda_{n_{k}}\right]}^{*}(\boldsymbol{x}, f \varepsilon) & =S_{\left[n_{k}, 2 \pi \lambda_{n_{k}}\right]}^{*}\left(\boldsymbol{x}, \sum_{i=1}^{\infty} f \varepsilon_{i}\right) \\
& \geqslant S_{\left[n_{k}, 2 \pi \lambda_{n_{k}}\right]}^{*}\left(\boldsymbol{x}, \sum_{i=1}^{n_{k}} f \varepsilon_{i}\right)-\sum_{i=n_{k}+1}^{\infty} S_{\left[0,2 \pi \lambda_{n_{k}}\right]}^{*}\left(\boldsymbol{x}, f \varepsilon_{i}\right) \geqslant n_{k}-\frac{1}{2^{n_{k}}} .
\end{aligned}
$$

Последнее вместе с (40) завершает доказательство теоремы 2.

Следующую лемму сформулируем без доказательства. Она наглядная и легко следует из геометрических соображений. 
ЛЕмма 5. Пусть $\Delta=\prod_{i=1}^{m}\left[a_{i}, b_{i}\right]$ есть замкнутый прямоугольник из $\mathbb{R}^{m}, a$ $\delta_{j}, \omega_{j} \subset \prod_{i=1}^{m}\left(a_{i}, b_{i}\right), j=1,2, \ldots, r,-$ семейство замкнутых кубов таких, что $\delta_{i} \cap \delta_{j}=\varnothing, \omega_{i} \cap \omega_{j}=\varnothing$ при $i \neq j$. Тогда существует гомеоморфизм $\tau: \Delta \rightarrow \Delta$, тохсдественный на граниче $\Delta, u \tau\left(\delta_{i}\right)=\omega_{i}, i=1,2, \ldots, r$.

Отсюда следует

ЛЕмма 6. Пусть функиия $f(\boldsymbol{x})$ непрерывна на $\Delta=\prod_{i=1}^{m}\left[a_{i}, b_{i}\right]$ и не имеет m-мерных интервалов постоянства. Тогда для любых чисел $\alpha_{i}, \beta_{i}, i=1,2, \ldots, r$, таких, что

$$
\inf _{\boldsymbol{x} \in \Delta} f(\boldsymbol{x}) \leqslant \alpha_{i}<\beta_{i} \leqslant \sup _{\boldsymbol{x} \in \Delta} f(\boldsymbol{x}), \quad i=1,2, \ldots, r
$$

и любого семейства попарно непересекающихся кубов $\delta_{j} \subset \prod_{i=1}^{m}\left(a_{i}, b_{i}\right), j=1, \ldots, r$, существует гомеоморфизм $\tau: \Delta \rightarrow \Delta$, тождественный на граниче $\Delta$, такой, что

$$
\alpha_{i}<f(\tau(\boldsymbol{x}))<\beta_{i}, \quad \boldsymbol{x} \in \delta_{i}, \quad i=1,2, \ldots, r .
$$

ДокАЗАТЕЛьство. Из (41) следует существование семейства попарно непересекающихся кубов $\omega_{i}$, содержашихся в $\prod_{i=1}^{m}\left(a_{i}, b_{i}\right)$, таких, что $\alpha_{i}<f(\boldsymbol{x})<\beta_{i}, \boldsymbol{x} \in \omega_{i}$, $i=1,2, \ldots, r$. Далее, применив лемму 5 , получим гомеоморфизм $\tau: \Delta \rightarrow \Delta \mathrm{c} \tau\left(\delta_{i}\right)=\omega_{i}$, для которого очевидно будет выполняться (42).

Обозначим через $\Gamma_{0}$ семейство всех гомеоморфизмов тора $\mathbb{T}^{m}$ и пусть $\Gamma_{N}=\left\{\tau \in \Gamma_{0}\right.$ : $\tau\left(\delta_{k}^{N}\right)=\delta_{k}^{N}, k \in I(N), \tau(\boldsymbol{x})$ тождественньй на границах $\left.\delta_{k}^{N}\right\}\left(\delta_{k}^{N}\right.$ определен в $\left.(2)\right)$.

Если $\boldsymbol{a}=\left(a_{1}, \ldots, a_{m}\right) \in \mathbb{R}^{m}$, то обозначим $\|\boldsymbol{a}\|=\max _{1 \leqslant i \leqslant m}\left|a_{i}\right|$.

Для любого $\tau \in \Gamma_{0}$ определим

$$
\omega(\delta, \tau)=\inf _{\left\|\boldsymbol{x}-\boldsymbol{x}^{\prime}\right\|>\delta}\left\|\tau(\boldsymbol{x})-\tau\left(\boldsymbol{x}^{\prime}\right)\right\| .
$$

ЛЕМма 7. Пусть $\left\{N_{k}\right\}_{k=1}^{\infty}$ есть возрастающая последовательность натуральных чисел таких, что $N_{k+1}$ делится на $N_{k}, a \sigma_{k} \in \Gamma_{N_{k}}, k=1,2, \ldots$, - последовательность гомеоморфизмов, причем для $\tau_{n}=\sigma_{n} \circ \sigma_{n-1} \circ \cdots \circ \sigma_{1}$ имеем

$$
\omega\left(\frac{1}{n}, \tau_{n}\right)>\frac{2}{N_{n+1}}, \quad n=1,2, \ldots .
$$

Тогда существует предел

$$
\tau(\boldsymbol{x})=\lim _{n \rightarrow \infty} \tau_{n}(\boldsymbol{x})
$$

и $\tau(\boldsymbol{x})$ представляет собой гомеоморфизм тора $\mathbb{T}^{m}$.

ДокАЗАТЕЛьСтво. Существование предела (45) и его непрерьвность очевидно следует из неравенства

$$
\left\|\tau_{n_{1}}(\boldsymbol{x})-\tau_{n}(\boldsymbol{x})\right\| \leqslant \frac{2 \pi}{N_{n}}, \quad \boldsymbol{x} \in \mathbb{T}_{m}, \quad n_{1}>n
$$

которое непосредственно вытекает из определения $\Gamma_{N}$. Нам следует только доказать, что $\tau(\boldsymbol{x})$ - взаимно-однозначное отображение. 
Если $\boldsymbol{x} \neq \boldsymbol{x}^{\prime}$, то $\left\|\boldsymbol{x}-\boldsymbol{x}^{\prime}\right\|>1 / n$ для некоторого натурального $n$. Тогда из (43) и (44) следует неравенство $\left\|\tau_{n}(\boldsymbol{x})-\tau_{n}\left(\boldsymbol{x}^{\prime}\right)\right\|>2 / N_{n+1}$, из которого имеем $\tau_{n}(\boldsymbol{x}) \in \delta_{\boldsymbol{i}}^{N_{n+1}}$, $\tau_{n}\left(\boldsymbol{x}^{\prime}\right) \in \delta_{i^{\prime}}^{N_{n+1}}, \overline{\delta_{\boldsymbol{i}}^{N_{n+1}}} \cap \overline{\delta_{i^{\prime}}^{N_{n+1}}}=\varnothing$, и, следовательно, $\tau(\boldsymbol{x}) \in \delta_{\boldsymbol{i}}^{N_{n+1}}, \tau\left(\boldsymbol{x}^{\prime}\right) \in \delta_{i^{\prime}}^{N_{n+1}}$. Отсюда же получаем $\tau(\boldsymbol{x})^{\prime} \neq \tau\left(\boldsymbol{x}^{\prime}\right)$. Теперь предположим, что $\boldsymbol{y} \in \mathbb{T}^{m}$ - любая точка. Имеем $\boldsymbol{y} \in \delta_{i_{k}}^{N_{k}}, k=1,2, \ldots$ Из (43) и (44) видно, что $\operatorname{diam} \tau^{-1}\left(\overline{\delta_{i_{k}}^{N_{k}}}\right)<1 / k$, $k=1,2, \ldots$. Отсюда следует, что

$$
\bigcap_{k=1}^{\infty} \tau^{-1}\left(\overline{\delta_{i_{k}}^{N_{k}}}\right)=\boldsymbol{x}, \quad \tau(\boldsymbol{x})=\boldsymbol{y} .
$$

Итак, доказано, что $\tau(\boldsymbol{x})$ есть гомеоморфизм.

ДОКАЗАТЕЛЬСТво ТЕОРЕМЫ 1 . Обозначим через $\delta^{N}(\alpha)$ семейство полуоткрытых кубов

$$
\delta_{i}^{N}(\alpha)=\left[\frac{2 \pi i_{1}}{N}, \frac{2 \pi\left(i_{1}+\alpha\right)}{N}\right) \times \cdots \times\left[\frac{2 \pi i_{m}}{N}, \frac{2 \pi\left(i_{m}+\alpha\right)}{N}\right), \quad i=\left(i_{1}, \ldots, i_{m}\right) \in I(N),
$$

где $0<\alpha \leqslant 1-$ некоторое число. Заметим, что при $\alpha=1$ это есть семейство $\delta^{N}$ (см. (2), (3)). Очевидно, можно предположить $|f(\boldsymbol{x})| \leqslant 1$ при $\boldsymbol{x} \in \mathbb{T}^{m}$.

В дальнейшем мы будем выбирать последовательности $N_{k} \rightarrow \infty, N_{k} \in \mathbb{N}, \alpha_{k} \rightarrow 1$, $0<\alpha_{k}<1, k=1,2, \ldots$, так, что $\alpha_{k}$ рационально и семейство $\delta^{N_{k+1}}$ разбивает $\delta^{N_{k}}\left(\alpha_{k}\right)$, т.е. каждое множество из семейств $\delta^{N_{k}}\left(\alpha_{k}\right)$ представимо в виде объединения кубов $\delta_{i}^{N_{k+1}}, i \in I\left(N_{k+1}\right)$.

Искомьй гомеоморфизм $\tau(\boldsymbol{x})$ получится в виде предела (45), где $\tau_{n}(\boldsymbol{x})=\sigma_{n} \circ \sigma_{n-1}$ 。 $\cdots \circ \sigma_{0}$ с $\sigma_{0} \in \Gamma_{0}, \sigma_{i} \in \Gamma_{N_{i}}, i=1,2, \ldots$, и будет выполняться неравенство (44).

Итак, нам предстоит построить последовательности $N_{k}, \alpha_{k}$ и $\sigma_{k}$ с изложенными выше свойствами, а также последовательность ступенчатых функций $Q_{k}(\boldsymbol{x})$ с интервалами постоянств $\delta_{\boldsymbol{i}}^{N_{k}}, \boldsymbol{i} \in I\left(N_{k}\right), k=1,2, \ldots$, такие, что

$$
\begin{gathered}
f\left(\tau_{n}(\boldsymbol{x})\right)=\sum_{i=1}^{n} Q_{i}(\boldsymbol{x})+R_{n}(\boldsymbol{x}), \\
S^{*}\left(\boldsymbol{x}, \sum_{i=1}^{n-1} Q_{i}\right) \leqslant A_{n}, \quad \boldsymbol{x} \in \mathbb{T}^{m}, \\
S_{\left[n, 2 \pi \lambda_{n}\right]}\left(\boldsymbol{x}, Q_{n}\right)>2 A_{n}, \quad \boldsymbol{x} \in G_{n},
\end{gathered}
$$

где

$$
\begin{gathered}
\left|R_{n}(\boldsymbol{x})\right| \leqslant 2^{n}, \quad x \in \mathbb{T}^{m}, \\
\mid R_{n}(\boldsymbol{x}) \leqslant\left(4 \pi \lambda_{n}+1\right)^{-m}, \quad \boldsymbol{x} \in \bigcup_{i \in I\left(N_{n}\right)} \delta_{i}^{N_{n}}\left(\alpha_{n}\right), \\
\left(2 \pi \alpha_{n}\right)^{m}>(2 \pi)^{m}-\frac{1}{2^{n}\left(4 \pi \lambda_{n}+1\right)^{m}},
\end{gathered}
$$


$A_{n} \rightarrow+\infty, \lambda_{n} \rightarrow+\infty$ - последовательности чисел, а $G_{n} \in \mathbb{T}^{m}, n=1,2, \ldots,-$ измеримые множества с

$$
\left|G_{n} \backslash \bigcup_{i=[\sqrt{n}]^{2}}^{n-1} G_{i}\right|>\frac{1}{2}\left|\mathbb{T}^{m} \backslash \bigcup_{i=[\sqrt{n}]^{2}}^{n-1} G_{i}\right|-\frac{1}{2^{n}}, \quad n \geqslant 1
$$

([ · ] есть целая часть). Построение будем вести по индукции. Начальный шаг тривиален. Предположим, что имеет место представление (46) с изложенньми выше соотношениями в случае $n=k$. Так как $\sum_{i=1}^{k} Q_{i}$ есть ступенчатая функция, то имеем

$$
S^{*}\left(x, \sum_{i=1}^{k} Q_{i}\right) \leqslant A_{k+1}, \quad x \in \mathbb{T}^{m}
$$

для некоторого числа $A_{k+1}>0$. Обозначим

$$
\mu_{k}=\inf _{\boldsymbol{i} \in I_{N_{k}}} \sup _{\boldsymbol{x} \in \delta_{\boldsymbol{i}}^{N_{k}}}\left|R_{k}(\boldsymbol{x})\right| .
$$

Из условия на функцию $f(\boldsymbol{x})$ и соотношения (46) следует, что $\mu_{k}>0$. Выберем $\lambda_{k+1}>\lambda_{k}$ такое, что

$$
\lambda_{k+1}>2^{k-2}(2 \pi)^{m-1}(k+1), \quad \frac{1}{2} \mu_{k} \xi\left(\frac{1}{2^{k-2}(2 \pi)^{m-1}}\right) \log \lambda_{k+1}>3 A_{k+1},
$$

где $\xi$ есть функция из леммы 1. При $\boldsymbol{x} \in K_{2^{-k-3}(2 \pi)^{1-m}}$ из (6) и (51) вытекает, что величина

$$
\max \left\{S_{\left[k+1,2 \pi \lambda_{k+1}\right]}^{*}\left(\boldsymbol{x}, \operatorname{Re} \varphi_{\lambda_{k+1}}\right), S_{\left[k+1,2 \pi \lambda_{k+1}\right]}^{*}\left(\boldsymbol{x}, \operatorname{Im} \varphi_{\lambda_{k+1}}\right)\right\}
$$

оценивается снизу через $3 A_{k+1} / \mu_{k}$. Обозначив

$$
\begin{aligned}
H^{\prime} & =\left\{\boldsymbol{x} \in \mathbb{T}^{m}: S_{\left[k+1,2 \pi \lambda_{k+1}\right]}^{*}\left(\boldsymbol{x}, \operatorname{Re} \varphi_{\lambda_{k+1}}\right)>\frac{3 A_{k+1}}{\mu_{k}}\right\}, \\
H^{\prime \prime} & =\left\{\boldsymbol{x} \in \mathbb{T}^{m}: S_{\left[k+1,2 \pi \lambda_{k+1}\right]}^{*}\left(\boldsymbol{x}, \operatorname{Im} \varphi_{\lambda_{k+1}}\right)>\frac{3 A_{k+1}}{\mu_{k}}\right\},
\end{aligned}
$$

имеем

$$
H^{\prime} \cup H^{\prime \prime} \supset K_{2^{-k-3}(2 \pi)^{1-m}}, \quad\left|K_{2^{-k-3}(2 \pi)^{1-m}}\right|>(2 \pi)^{m}-\frac{1}{2^{k+1}} .
$$

Отсюда следует, что для одного из двух множеств $H^{\prime}$ и $H^{\prime \prime}$, которое обозначим через $G_{k+1}$, имеет место неравенство

$$
\left|G_{k+1} \backslash \bigcup_{i=[\sqrt{k+1}]^{2}}^{k} G_{i}\right|>\frac{1}{2}\left|\mathbb{T}^{m} \backslash \bigcup_{i=[\sqrt{k+1}]^{2}}^{k} G_{i}\right|-\frac{1}{2^{k+2}}
$$

Обозначим через $\psi_{k+1}(\boldsymbol{x})$ ту из функций $\mu_{k} \operatorname{Re} \varphi_{\lambda_{k+1}}$ или $\mu_{k} \operatorname{Im} \varphi_{\lambda_{k+1}}$, которая естественньм образом связана с $G_{k+1}$. Итак, имеем

$$
G_{k+1}=\left\{\boldsymbol{x} \in \mathbb{T}^{m}: S_{\left[k+1,2 \pi \lambda_{k+1}\right]}^{*}\left(\boldsymbol{x}, \psi_{k+1}\right)>3 A_{k+1}\right\}
$$


Далее, учитьвая рациональность числа $\alpha_{k}$, можно выбрать $N_{k+1}$ так, чтобы семейство $\delta^{N_{k+1}}$ разбивало $\delta^{N_{k}}\left(\alpha_{k}\right)$ и выполнялось неравенство

$$
\sup _{\boldsymbol{i} \in I_{N_{k+1}}} \sup _{\boldsymbol{x}^{\prime}, \boldsymbol{x}^{\prime \prime} \in \delta_{i}^{N_{k+1}}}\left|\psi_{k+1}\left(\boldsymbol{x}^{\prime}\right)-\psi_{k+1}\left(\boldsymbol{x}^{\prime \prime}\right)\right| \leqslant \frac{1}{\left(4 \pi \lambda_{k+1}+1\right)^{m}} .
$$

Пусть $\alpha_{k+1}$ такое рациональное число, что

$$
\left(1-\frac{1}{2^{k+1}\left(4 \pi \lambda_{k+1}+1\right)^{m}}\right)^{1 / m}<\alpha_{k+1}<1 .
$$

Из (46) следует, что $R_{k}(\boldsymbol{x})$ непостоянна ни на каком интервале. Отсюда, применив лемму 6 в каждом интервале $\delta_{i}^{N_{k}}$, получим такой гомеоморфизм $\sigma_{k+1} \in \Gamma_{k+1}$, что

$$
\begin{aligned}
\inf _{\boldsymbol{x} \in \delta_{\boldsymbol{i}}^{N_{k+1}\left(\alpha_{k+1}\right)} \psi_{k+1}(\boldsymbol{x})} & \inf _{\boldsymbol{x} \in \delta_{\boldsymbol{i}}^{N_{k+1}}\left(\alpha_{k+1}\right)} R_{k}\left(\sigma_{k+1}(\boldsymbol{x})\right) \leqslant \sup _{\boldsymbol{x} \in \delta_{\boldsymbol{i}}^{N_{k+1}}\left(\alpha_{k+1}\right)} R_{k}\left(\sigma_{k+1}(\boldsymbol{x})\right) \\
& \leqslant \sup _{\boldsymbol{x} \in \delta_{\boldsymbol{i}}^{N_{k+1}}\left(\alpha_{k+1}\right)} \psi_{k+1}(\boldsymbol{x}) .
\end{aligned}
$$

Определим

$$
\begin{gathered}
Q_{k+1}(\boldsymbol{x})=\psi_{k+1}\left(\operatorname{centre} \delta_{i}^{N_{k+1}}\right) \quad \text { при } \boldsymbol{x} \in \delta_{i}^{N_{k+1}}, \\
R_{k+1}(\boldsymbol{x})=R_{k}\left(\sigma_{k+1}(\boldsymbol{x})\right)-Q_{k+1}(\boldsymbol{x}) .
\end{gathered}
$$

Из (53)-(55) следует, что

$$
\left|\psi_{k+1}(\boldsymbol{x})-Q_{k+1}(\boldsymbol{x})\right| \leqslant \frac{1}{\left(4 \pi \lambda_{k+1}+1\right)^{m}}, \quad \boldsymbol{x} \in \mathbb{T}^{m},
$$

а также условия $(48),(49)$ при $n=k+1$, и поэтому с учетом $(52)$ при $\boldsymbol{x} \in G_{k+1}$ получаем

$$
S_{\left[k+1,2 \pi \lambda_{k+1}\right]}^{*}\left(x, Q_{k+1}\right) \geqslant S_{\left[k+1,2 \pi \lambda_{k+1}\right]}^{*}\left(x, \psi_{k+1}\right)-1 \geqslant 3 A_{k+1}-1>2 A_{k+1} \text {. }
$$

Таким образом, построена последовательность гомеоморфизмов $\left\{\sigma_{n}\right\}_{n=1}^{\infty}, \sigma_{n} \in \Gamma_{n}$, со свойствами (46)-(50). Докажем, что предел $\tau(\boldsymbol{x})$ (см. (45)) является искомьм гомеоморфизмом. Во-первых, в силу (46) заметим, что

$$
f(\tau(\boldsymbol{x}))=\sum_{k=1}^{n} Q_{k}(\boldsymbol{x})+P_{n}(\boldsymbol{x})
$$

где

$$
P_{n}(\boldsymbol{x})=R_{n}\left(\sigma_{n+1}\left(\sigma_{n+2}(\ldots(\boldsymbol{x}) \ldots)\right)\right) .
$$

Очевидно, что

$$
\begin{gathered}
\left|P_{n}(\boldsymbol{x})\right| \leqslant 2^{n}, \quad x \in \mathbb{T}^{m} \\
\left|P_{n}(\boldsymbol{x})\right| \leqslant \frac{1}{\left(4 \pi \lambda_{n}+1\right)^{m}}, \quad x \in \bigcup_{i \in I\left(N_{n}\right)} \delta_{i}^{N_{n}}\left(\alpha_{n}\right) .
\end{gathered}
$$


С другой стороны, из (49) и определения $\delta_{i}^{N}(\alpha)$ имеем

$$
\left|\bigcup_{i \in I\left(N_{n}\right)} \delta_{i}^{N_{n}}\left(\alpha_{n}\right)\right| \geqslant(2 \pi)^{m}-\frac{1}{2^{n}\left(4 \pi \lambda_{n}+1\right)^{m}}
$$

Из (57), (58) вытекает неравенство

$$
S_{\left[0,2 \pi \lambda_{n}\right]}^{*}\left(\boldsymbol{x}, P_{n}\right) \leqslant 2, \quad \boldsymbol{x} \in \mathbb{T}^{m} .
$$

Так же, как и при доказательстве теоремы 2 (см. (38)-(40)), с учетом (50) можно убедиться, что множество $G=\underline{\lim }_{n \rightarrow \infty} G_{n}$ имеет полную меру. С другой стороны, если $\boldsymbol{x} \in G$, то $\boldsymbol{x} \in G_{k_{n}}$ для некоторой последовательности $k_{n} \rightarrow \infty$. Из (47), (56) и (59) имеем

$$
\begin{aligned}
S_{\left[k_{n}, 2 \pi \lambda_{k_{n}}\right]}^{*}(\boldsymbol{x}, f(\tau(\boldsymbol{x}))) & \geqslant S_{\left[k_{n}, 2 \pi \lambda_{k_{n}}\right]}^{*}\left(\boldsymbol{x}, Q_{k_{n}}\right)-S^{*}\left(\boldsymbol{x}, \sum_{i=1}^{k_{n}-1} Q_{i}\right)-S_{\left[0,2 \pi \lambda_{\left.k_{n}\right]}\right]}^{*}\left(\boldsymbol{x}, P_{k_{n}}\right) \\
& \geqslant 2 A_{k_{n}}-A_{k_{n}}-2>A_{k_{n}}-2 .
\end{aligned}
$$

Итак, теорема 1 доказана.

\section{СПИСОК ЦИТИРОВАННОЙ ЛИТЕРАТУРЫ}

[1] Олевский А. М. Модификации функций и ряды Фурье // УМН. 1984. Т. 40. № 3. С. 157-193.

[2] Bohr H. Über einen Satz von J. Pal // Acta Sci. Math. (Szeged). 1935. V. 7. P. 129-135.

[3] Саакян А. А. Интегральные модули гладкости и коэффициенты Фурье у суперпозиции функции // Матем. сб. 1979. Т. 110. № 4. С. 597-608.

[4] Kahane J.-P., Kaznelson Y. Series de Fourier des functions bornées // Studia Pure Math. / ed. P. Erdös. Mem. Paul Turan. Budapest: Akad. Kiadó, 1983. P. 395-410.

[5] Fefferman Ch. On the divergence of multiple Fourier series // Bull. Amer. Math. Soc. 1971. V. 77. № 2. P. 191-195. 